\title{
Caractérisation des sols pour le tunnel de la rocade nord de Grenoble
}

J. MONNET

LIRIGM

Université Joseph-Fourier BP 53

38041 Grenoble Jmonnet@ujf-grenoble.fr

\section{CHAPEAU}

CETE-Lyon

25, av. François-Mitterrand

Case 1

69674 Bron Cedex Christian.Chapeau@ equipement.gouv.fr

G. GODARD

DDE,

17, bd Joseph-Vallier 38000 Grenoble

Gilles.Godard@ equipement.gouv.fr

La construction de tunnel en site urbain est un problème particulièrement délicat par le contrôle soigneux à la fois des règles de sécurité à appliquer, pour prévenir la rupture, pour maitriser les déplacements de surface et pour ne pas nuire au bâti existant. Au niveau d'un avant-projet, il s'agit d'évaluer les variantes fonctionnelles d'un point de vue technico-économique et de proposer la bonne adaptation de Youvrage à la nature géotechnique des sols. En particulier la connaissance, dès ce stade, des caractéristicques géomécaniques du sol s'est avérée primordiale. Le tracé préliminaire du tunnel de la future rocade nord de Grenoble démarre du quartier des Sablons, traverse en sous-fluvial 1'Tsère, passe sous le quartier de ITle verte, franchit une seconde fois l'Isère pour rejoindre l'autoroute existante A48 à la Porte de France, La géologie du tracé comporte une partie de terrains d'origine alluviale avec des sables limoneux et des argiles, puis une traversée dans les calcaires de Chartreuse dès le second franchissement de l'Isère. La méthode de reconnaissance choisie utilise des forages pour avoir un accès direct au sol environnant l'ouvrage qui se trouve entre 10 et $20 \mathrm{~m}$ de profondeur pour décrire les sols, les classer et étudier leur comportement lors la réalisation des ouvrages projetés. Pour les sables limoneux sous la nappe phréatique qui sont difficiles à prélever, on a privilégié la recherche des paramètres de déformation et de rupture par essais pressiométriques, respectant mieux leur structure. La variabilité des données géotechniques a été prise en compte dans le choix des paramètres géomécaniques du projet et des solutions pour le creusement du tunnel. Le projet devrait voir le jour dans la première décennie de ce nouveau siècle.

Mots-clés : essais in situ et mesures, tunnel, pressiomètre, angle de frottement.

\section{Soil characterisation for the north transversal road on grenoble area}

Tunnel construction in urban area is a particular sensitive problem due to the careful security requirements, which must be applied to prevent failure, and due to the displacements, which must be as small as possible to avoid damage to old buildings. For the preliminary design, it is necessary to study each alternative plan of the project and to propose the best adaptation of the construction to the soil. The determination of the geomechanical characteristics is essential.

The preliminary layout for the North transversal road tunnel of Grenoble begins at the Sablon district, crosses under the Isere river, passes under the a lie Verte $\mathrm{x}$ district, crosses Isère a second time to join the motorway A48 at Porte de France. The geology of the layout includes in its first part alluvial soil with silty sands and clays, then after the second crossing of the Isere river it includes the Chartreuse fimestones. The selected geotechnical investigation uses boreholes to study the soll, at the level of the construction that is 10 to $20 \mathrm{~m}$ deep, for soil description, classification, and behaviour along the tunnel construction. Deformation and failure parameters are measured by pressuremeter tests because sampling of silty sands under the water table is very difficult. The data variability of the geomechanical parameters is taken into account for the project and for the tunnel drilling.

The project should be constructed in the first decade of this new century.

Key words : field testing and monitoring, tunnel, pressuremeter, friction angle. 


\section{NOTATIONS}

a

b

C

G

$E_{M}$

$E_{M}$

E

E'

$\alpha$

Y

$\mathrm{K}_{0}$

$\phi^{\prime}$

$\phi_{\mu}$

$\psi$

$\mathrm{P}_{0}$

$P_{1}$

$\frac{P_{1}}{P_{1}}$

$P_{\text {loaicalc : }}$ pression limite théorique

$\sigma_{n} \quad$ : écart-type de la loi normale d'un

$\sigma_{r}^{\prime}, \sigma_{\theta}^{\prime} \sigma_{x}^{\prime}$ : contraintes effectives radiales,

circonférentielles, verticales

$\sigma_{\text {tb }}$

$\varepsilon_{p}, \varepsilon_{p}$

$r_{0}$

$V_{1}$

X échantillon de mesures

rayon du forage

rayon externe de la première zone plastique plastique

déplacement radial au contact du forage

appliquée par le pressiomètre

diametre de la plus grosse particule de l'échantillon

module élastique de cisaillement

module pressiométrique Ménard la

norme NF 94-110-1

module pressiométrique Ménard

corrigé en épaisseur et déformée de sonde

norme NF 94-110-2

rapport $\mathrm{E}_{\mathrm{M}} / \mathrm{E}$

poids volumique du sol

coefficient de pression des terres au repos

angle de frottement interne

angle de frottement intergranulaire

angle de dilatance

pression horizontale des terres au repos

selon la norme NF 94-110-1

pression limite nette égale à $\mathrm{p}_{1}-\mathrm{p}_{\mathrm{i}}$

pression limite conventionnelle

corrigée en épaisseur et déformée

contrainte effective radiale au niveau

du rayon b

déformations radiales

et circonférentielles

rayon initial du forage

volume initial de la sonde

pressiométrique

volume injecté dans la sonde

pressiométrique pour recompacter

le sol à l'état initial mesure rayon externe de la seconde zone

valeur moyenne d'un échantillon de

\section{Introduction}

Le projet «tunnel sous la Bastille » a été inscrit au schéma directeur de 1973 mais sa réalisation était envisagée après celle de la rocade sud de Grenoble. La
Saturation rapide des grandes infrastructures de l'agglomération au cours des années 80 , le succès des deux lignes de tramway mises en service en 1987 et 1990 ont relancé le débat sur l'organisation des transports. Celui-ci s'est concrétisé par le Plan de déplacements urbains (PDU), et la révision du schéma directeur en 2000. Ces différentes réflexions ont progressivement et fondamentalement modifié le projet en l'intégrant dans une stratégie globale et multimodale d'organisation des déplacements. L'État a engagé, dès 1996, des études d'opportunité de la rocade nord ainsi que des études techniques. Les études d'opportunité ont mis en évidence l'utilité et la forte rentabilité du projet ainsi que la nécessité de poursuivre les études techniques.

Le Service " grands travaux " de la DDE de l'Tsère a confié au LRPC région Rhône-Alpes du CETE de Lyon la mission de reconnaissance spécifique du site pour la mise au point du programme, la préparation des pièces techniques de la consultation des entreprises de sondages, le suivi de la campagne, la réalisation d'essais et la synthèse géotechnique.

La construction de tunnel en site urbain est un problème particulièrement délicat par le contrôle soigneux à la fois des règles de sécurité à appliquer pour prévenir la rupture et maîtriser les déplacements de surface pour ne pas nuire au bâti existant. Du point de vue techinique et économique, le projet doit être conçu et dimensionné en s'adaptant aux contraintes du sous-sol qu'il faut bien reconnaître et connaître. Pour cela, la méthode usuelle d'investigation est de prélever des échantillons de sol non remaniés qui seront ensuite testés au laboratoire. Malheureusement, les sols, le plus souvent sableux, rencontrés dans la plaine de l'Isère sont situés sous la nappe phréatique et difficiles à prélever. Ils se désagrègent dès la sortie du carottier. L'analyse pressiométrique est alors intéressante, car elle permet de caractériser de tels sols en place en respectant leur structure naturelle, dans la mesure où le forage préalable ne remanie pas le sol. Celle-ci a été privilégiée dans la reconnaissance du site.

\section{2}

\section{Description du projet}

\section{1}

\section{Cadre du Plan de déplacements urbains (PDU)}

La rocade nord (Fig. 1) terminera l'anneau (longueur $21 \mathrm{~km}$ ) qui trace grossièrement un cercle inscrit de l'ordre de $5 \mathrm{~km}$ de diamètre autour de Grenoble avec 15 diffuseurs et qui boucle la ville. Cet anneau est une pièce maîtresse de la stratégie du plan de déplacements urbains qui vise à limiter l'usage de la voiture particulière dans les zones urbaines denses, au profit des piétons, de cycles et des transports en commun. Les échangeurs de la rocade nord permettent d'ailleurs une desserte efficace des futurs parcs relais (parcs de stationnement attachés aux grandes stations d'interconnexion du réseau de transports en commun) du secteur nord.

Les prévisions de trafic conduisent à une moyenne de 60000 véhicules/jour, dont un peu moins de la moitié utilise la rocade Nord pour relier l'amont et l'aval de 1'Isère. L'ouverture au PL de la rocade nord permettrait 
de transférer environ $4000 \mathrm{PL}$ /jour sur ce nouvel itinéraire, mais il renchérit le coût de l'ouvrage de l'ordre de 30 à $50 \%$ et ne permet pas de maintenir le diffuseur de 1'hôpital Michallon pour des raisons de rampe. L'interdiction aux poids lourds permet également de réduire significativement à la source les risques d'accidents dans le tunnel. L'importance du surcoût de l'ouverture au PL, comparé aux bénéfices qui peuvent être attendus, conduit à proposer un projet à accès limité, communément appelé à " gabarit réduit).

Les différentes options techniques (ou caractéristiques de l'ouvrage) évoquées dans le PDU ont fait. l'objet d'examens spécifiques et ont conduit à l'élaboration de quatre familles de tracé (Fig. 2) susceptibles de répondre au cahier des charges. Une analyse exhaustive des variantes conduit à retenir la famille «POS $»$ qui passe sous le coeur de "l'Tle verte » pour des raisons de fiabilité des techniques de construction, de coùt, de fonctionnalité et d'impact du chantier et du projet. Dans cette dernière famille, l'étude a été approfondie sur des variantes qui se distinguent par la définition du gabarit des véhicules admissibles ou par le profil en long du projet. L'étude a porté sur les gabarits admissibles et de deux variantes (Fig. 3 ) de profil en long

- variante «Esplanade » qui traverse l'Ile verte et le site de la Bastille en tunnel, franchit l'Tsère par un viaduc, emprunte le passage de la rue Durand-Savoyat, franchit le faisceau des voies ferrées et traverse le site du Centre d'études nucléaires de Grenoble (CENG) en tranchée couverte;

- variante "Vercors $»$ qui traverse ITle verte, le site de la Bastille et la presqu'île en tunnel.

Les tunnels à " gabarit réduit » sont normalisés selon trois hauteurs standards (Fig. 4), 2 m, 2,7 m et $3,5 \mathrm{~m}$. Leurs avantages sont nombreux, augmentation significative des pentes du tunnel et des trémies d'accès, amélioration de l'intégration du projet dans un environnement contraint et plus précisément íci possibilité de réaliser un échangeur au niveau de l'hôpital Michallon, intégration plus facile de la tranchée couverte de la presqu'île, coût plus faible, mais surtout transport interdit aux camions donc une sécurité accrue par la suppression du risque de très grave incendie lié à la circulation PL. Par contre, ils présentent trois contraintes, les fumées ne peuvent pas rester stratifiées sous le plafond, les véhicules de secours doivent être adaptés et un tri des véhicules à l'entrée doit ẻtre opẻré.

Le coùt total du projet est estimé entre $378 \mathrm{M} € \mathrm{HT}$ et 509 M€ HT selon les variantes.

\section{2}

\section{Les choix techniques de gabarit et de nombre de tubes}

Le « gabarit réduit » peut ètre modulé selon trois hauteurs standard:

- gabarit de $2 \mathrm{~m}$. Ce gabarit permet le passage de $93 \%$ des véhicules intéressés par le projet:

- gabarit de $2,7 \mathrm{~m}$. Ce gabarit permet le passage de $96 \%$ des véhicules intéressés par le projet. L'exploitant doit prévoir l'acquisition et la maintenance d'un matériel spécifique d'intervention et de lutte contre l'incendie. Ce gabarit coûte environ $15 \%$ de plus que le gabarit de $2 \mathrm{~m}$ et lui semble préférable pour des raisons de sécurité :

- gabarit de $3,5 \mathrm{~m}$. Ce gabarit permet le passage de $98,5 \%$ des véhicules intéressés par le projet. Ce gabarit augmente les coûts de $30 \%$ de plus que le gabarit de $2,7 \mathrm{~m}$. Il n'a pas été retenu.

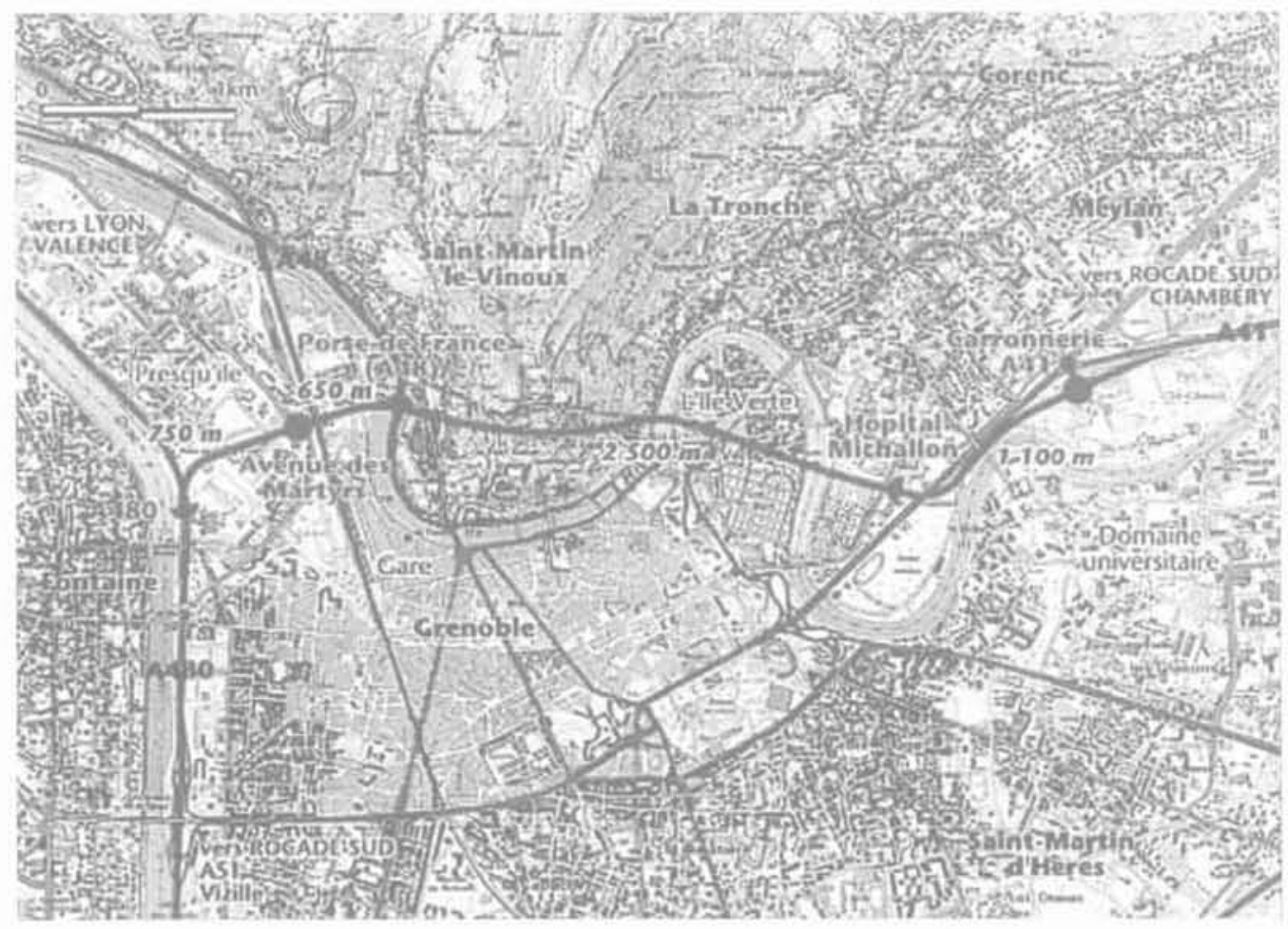

FG.1 La vue en plan du projet de la rocade nord de Grenoble.

The plane view of the North transversal road of Grenoble. 


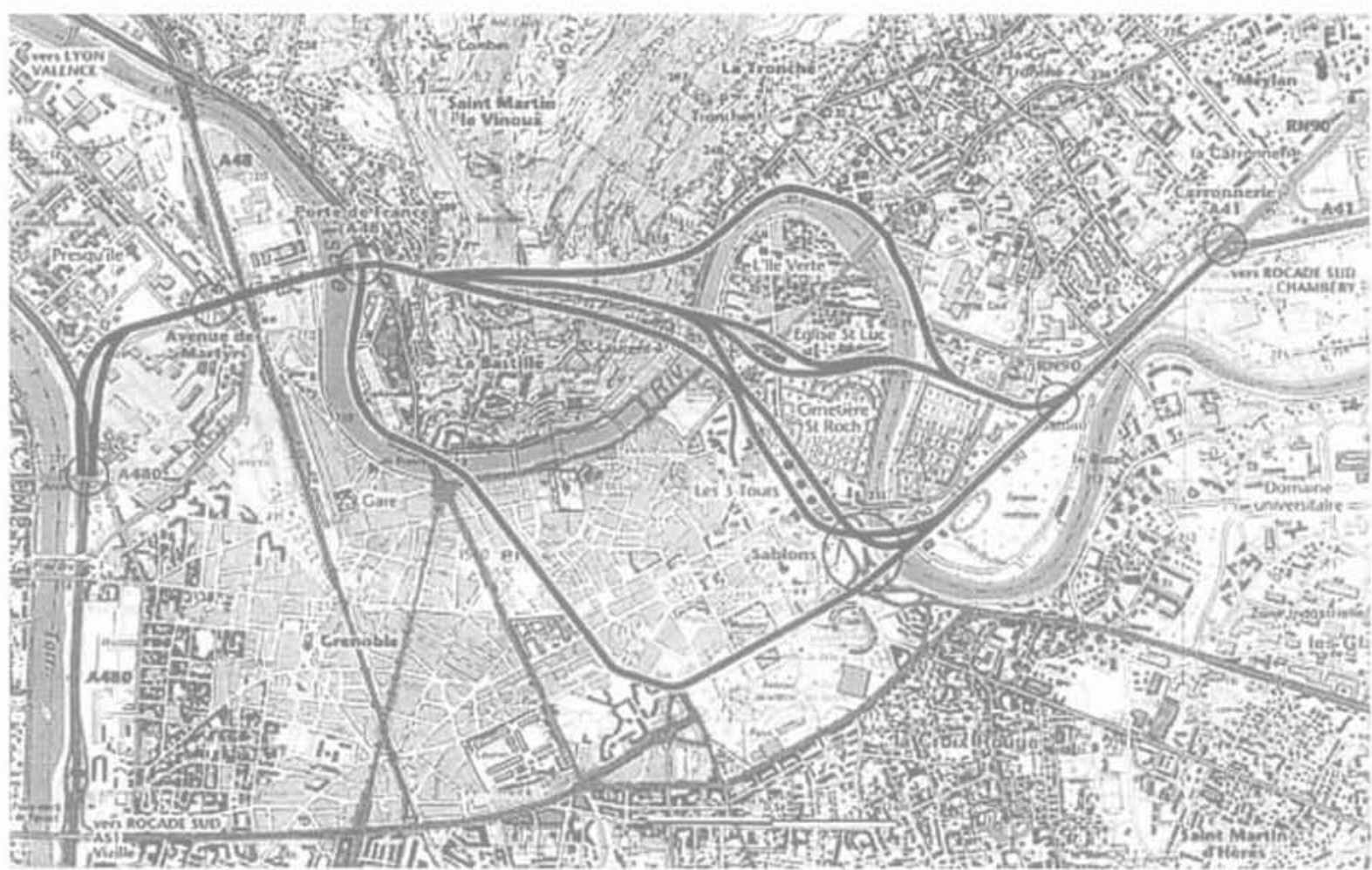

FIG. 2 Les quatre familles: «Ville $», ~ «$ Leclerc $», ~ «$ Quai $», ~ « P O S »$.

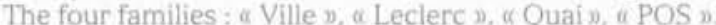

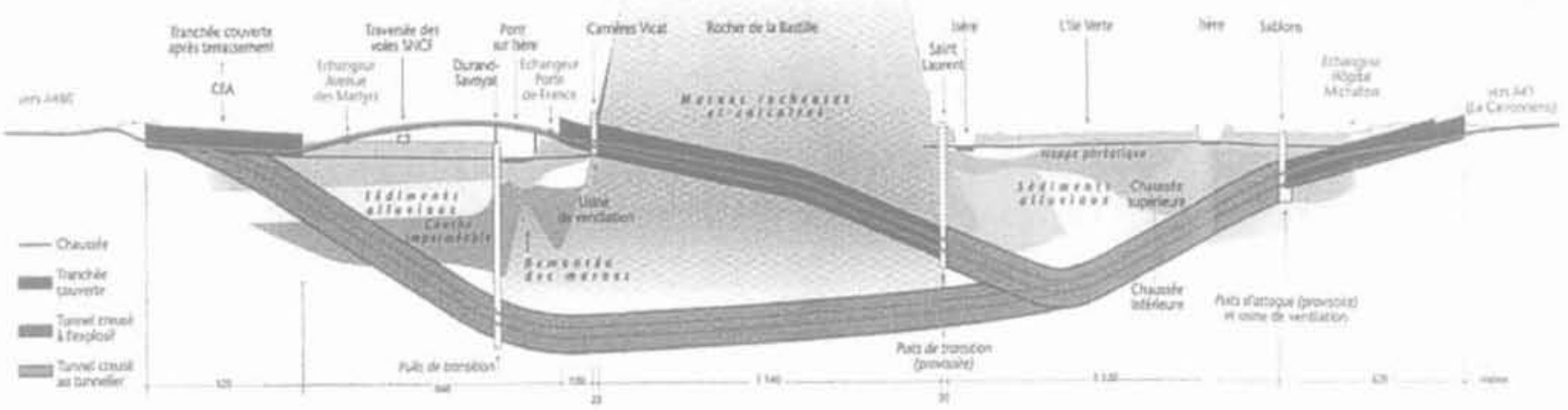

FG. 3 Les variantes « Esplanade $n$ et «Vercors $»$ de profils en long de la famille $«$ POS $»$.

The two $\alpha$ Esplanade $n$ and "Vercors $n$ longitudinal profile of the $\alpha$ POS $n$ families.

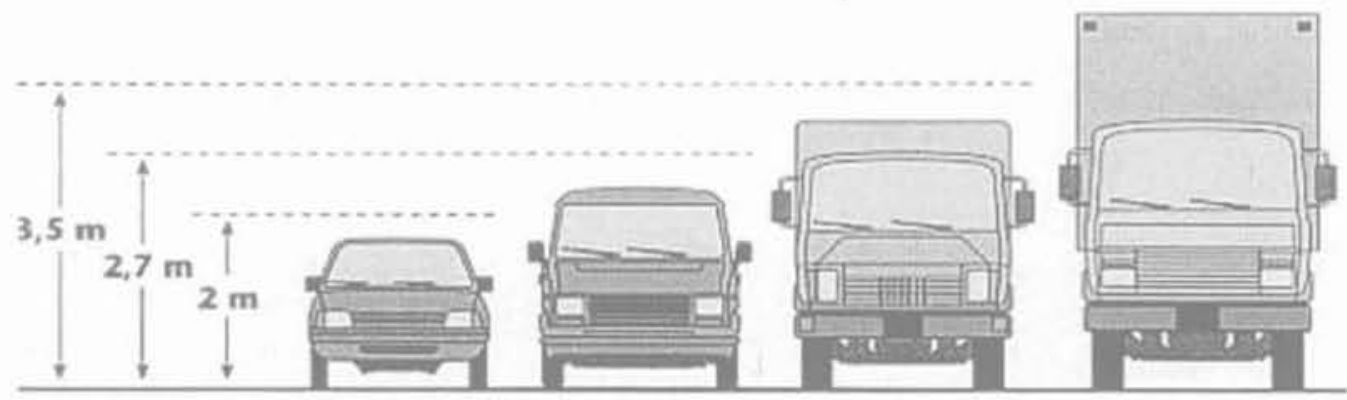

FG. 4 Les types de véhicules standard. 
Les profils en travers possibles ont été examinés en fonction des gabarits admissibles, de la vitesse de référence et du niveau d'exploitation retenu. Dans le cas du gabarit de $2 \mathrm{~m}$, la superposition des chaussées permet d'optimiser les différents espaces générés par le tunnel sans que son diamètre soit excessif, ce qui n'est pas le cas des tubes juxtaposés qui génère des espaces inutilisés. Par contre, il est nécessaire de réaliser régulièrement des ouvrages d'interconnexion entre les niveaux pour assurer le passage des usagers et des secours d'une chaussée à l'autre. L'analyse du coût giobal est alors favorable à un profil monotube pour le gabarit de $2 \mathrm{~m}$. Dans le cas d'un gabarit admissible de $2,7 \mathrm{~m}$, la superposition des chaussées dans un tunnel creusé conduit à un diamètre important et engendre des espaces inutilisés dans la section. La juxtaposition des chaussées dans deux tubes séparés permet une utilisation plus efficace des espaces creusés, une réduction du diamètre du tunnel et une meilleure rentabilisation de son utilisation. Ces considérations, couplées à la géologie rencontrée par le projet conduisent à des coûts pratiquement comparables pour les deux types de profils. Dans la mesure où la juxtaposition des chaussées offre plus de souplesse à l'exploitant, un profil à deux tubes séparés (Fig. 5) reliés régulièrement par des rameaux a été retenu pour un gabarit de $2,7 \mathrm{~m}$.

3

\section{Description du sous-sol de la rocade nord}

\section{1}

\section{Cadre géologique général}

Le projet de la rocade nord de Grenoble rencontre deux formations géologiques aux origines très distinctes :

- les alluvions de la vallée de l'Isère d'épaisseur très importante qui peut varier entre 500 et $800 \mathrm{~m}$. Ce sol est constitué de graves, sables, limons et argiles d'origine quaternaire. Les dépôts sont d'origine lacustre lors de la dernière période de glaciation mais aussi fluviale. A noter que les formations alluviales hètérogènes dans lesquelles les tunnels sont envisagés sont susceptibles de contenir des blocs de forte taille provenant de cônes de déjection ou d'éboulis anciens ainsi que d'éventuels troncs d'arbres flottés, sédimentés avec les alluvions. Il faut surtout souligner l'existence très pro-

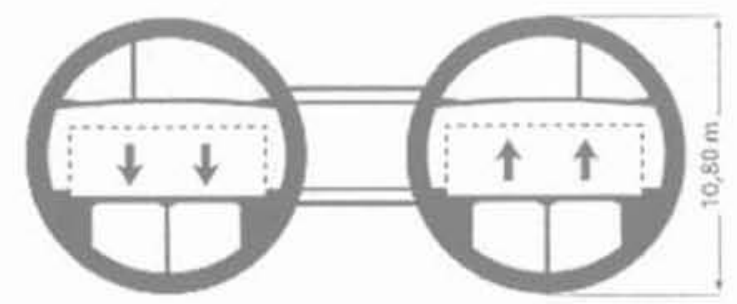

FG,5 La section type du projet de la rocade nord de Grenoble.

The chosen section of the North transversal tunnel of Grenoble. bable de nombreux paléochenaux qui peuvent fournir localement des changements brutaux de faciès (sols fins dans un dépôt alluvionnaire plus grossier, par exemple) :

- les formations rocheuses sédimentaires des contreforts du massif de Chartreuse sous la Bastille. Ces roches datent du Jurassique supérieur et Crétacé inférieur et font apparaitre une alternance de bancs calcaires et de bancs marneux. Les strates de ces roches présentent un pendage subvertical. La roche est recoupée par trois failles principales dont le remplissage broyé et altéré peut présenter des circulations d'eau.

\section{2}

\section{Description géotechnique des terrains}

La campagne de reconnaissance s'est concentrée en 1996-1997 sur la traversée de l'lle verte, point dur du projet, avec passage en tunnel sous le bâti. On rapporte ici les résultats de cette campagne et des essais réalisés ainsi que la démarche permettant d'orienter les choix techniques. La synthèse géotechnique permet de proposer à partir des sondages de 20 à 40 m de profondeur effectués sur le tracé un classement des terrains rencontrés en neuf familles de sols ou de roches, suivant leurs origines de dépôt, leurs localisations et leurs caractéristiques :

- famille F1 : des remblais de surface localement 1 à $4 \mathrm{~m}$ d'épaisseur:

- famille F2 : des argiles plastiques sous-jacentes à la couche F1 de faible épaisseur ;

- famille F3 : des limons sableux marron à verts de surface sur 3 à $7 \mathrm{~m}$ d'épaisseur localement sous-jacents aux remblais F1 et surmontant les sables et graviers ;

- famille F4 : des sables et graviers généralement constitués de sables fins gris noirâtres parfois vaseux (sablons) à passées limoneuses ou sablo-graveleuses (vers l'est) de puissance 3 à $20 \mathrm{~m}$ et qui sont sousjacents aux limons F3. Les graviers excèdent rarement $100 \mathrm{~mm}$ et leur diamètre maximal est le plus souvent $20 \mathrm{~mm}$. Ils sont d'origine alluvio-fluviale ;

- famille F5 : des limons argileux gris remplaçant les sables et graviers à l'est :

- famille F6 : des sables fins et limons en alternance gris noirs moyennement compacts se situant sous les sables et graviers de la famille F4 avec des passées limoneuses, voire argileuses :

- famille F7: des limons sableux gris noir profond peu compacts se retrouvant à l'ouest du projet, se déstructurant au prélèvement. D'origine glacio-lacustre ou fluviatile, ils sont cependant traités globalement, leurs caractéristiques étant homogènes ;

- famille F8: des sables profonds compacts (sablons) situés très profondément. Ce sable se rencontre à un niveau profond principalement côté Sablons, à l'est à des profondeurs décroissantes en allant de l'ouest vers l'est. Ce sable est décrit à partir des forages destructifs comme un sable fin gris avec quelques graviers comme le sol F6 mais avec des caractéristiques pressiométriques beaucoup plus élevées :

- famille F9 : des marnes rocheuses et calcaires constituant le rocher de la Bastille.

La coupe géologique le long du profil du projet est présentée sur la figure 3. 


\section{Les reconnaissances géotechniques}

Les reconnaissances géotechniques se sont déroulées en plusieurs phases successives avec les moyens suivants :

- 4 sondages destructifs avec enregistrement de paramètres, sur le quai $X$-Jouquin en rive droite de I'Tsère pour préciser le toit du rocher :

- 8 sondages carottés, de profondeur 30 à $40 \mathrm{~m}$ le long du tracé pour prélèvement d'échantillons et essais de laboratoire :

- 15 sondages pressiométriques standard et avec cycles de charge-décharge, de profondeur 30 à $40 \mathrm{~m}$, le long du tracé.

Les essais de laboratoire ont complété les essais de reconnaissance par ; des essais d'identification physique, des essais cedométriques, des essais triaxiaux CD avec mesure des variations de volume, des essais triaxiaux cycliques. Les résultats des essais in situ sont incliqués dans le tableau I. Nous ne détaillerons pas ici l'ensemble des résultats des essais in situ et en laboratoire, et nous nous attacherons principalement à la problématique posée par l'évaluation des caractéristiques de cisaillement des sols limono-sableux constitués par les dépôts fluvioglaciaires. Il a été décidé de privilégier l'essai pressiométrique pour les raisons suivantes : c'est un essai in situ quî teste le sol dans son état naturel dans la mesure où le forage préalable ne remanie pas le sol, il est réalisable même dans les sols pulvérulents grossiers; il peut être positionné en XYZ et il permet de déterminer le module élastique de cisaillement G et l'angle de frottement interne $\phi^{\prime}$ (ou la cohésion non drainée $c_{u}$ ) au niveau du projet.

\section{4}

\section{Utilisations de l'essai pressiométrique}

\section{1}

\section{L'essai pressiométrique normalisé}

L'essai pressiométrique (Ménard, 1955) est utilisé largement a ce jour pour le dimensionnement des fondations (Ménard, 1957; Gambin, 1979; Amar et al., 1991; Fascicule 62 titre V, 1993) à partir du module pressiométrique $\mathrm{E}_{\mathrm{M}}$ et de la pression limite $\mathrm{p}_{i}$ (Norme NF P
94-110-1). Quand le sol est granulaire, il peut être utilisé pour estimer l'angle de frottement du sol, par corrélation selon des expériences sur les sables (Ménard, 1957).

Nous utilisons l'essai pressiométrique comme un essai de cisaillement en place (Clarke et al., 1998) permettant d'obtenir le module élastique de cisaillement et l'angle de frottement interne quand le sol est granulaire. Ces valeurs peuvent être utilisées pour décrire l'état de compacité des sols, pour la conception et l'approche dimensionnelle des ouvrages du tunnel de la Rocade nord. Les paramètres de comportement mécaniques du sol permettent alors d'adapter l'ouvrage en terrains meubles à son environnement géotechnique et d'optimiser le projet.

\section{2}

\section{Théorie élastoplastique du cisaillement autour du pressiomètre}

Nous ne détaillerons pas ici l'ensemble de la théorie élastoplastique du cisaillement du sol autour du pressiomètre, et nous renverrons le lecteur à la référence bibliographique Monnet (1990), Monnet et Khlif (1994) mais nous indiquerons seulement ses principales hypothèses et conclusions. L'intérêt de cette démonstration est qu'elle fournit un cadre plus rigoureux à une interprétation de l'essai pressiométrique en cisaillement, sans nécessiter le passage par des corrélations toujours délicates à manipuler et dont on connait mal les limites d'utilisation.

\section{Hypothèses}

Le sol a un comportement élastique linéaire de module $\mathrm{G}$ avec une plasticité non associée d'angle de frottement interne $\phi^{\prime}$, d'un angle de clilatance $\Psi$ lié au frottement intergranulaire $\phi_{\mu}$ (Monnet et Gielly, 1978) par:

$$
\psi=\phi^{\prime}-\phi_{\mu}
$$

Si l'angle de frottement interne d'un sol varie inversement proportionnellement à l'indice des vides et peut prendre des valeurs différentes selon l'état de densité du sol, par contre sa valeur est bornée par un minimurn qui est l'angle de frottement intergranulaire. Dans ce cas le sol n'exprime plus aucune dilatance.

Résultats de la campagne de reconnaissance géotechnique in situ.

Results of the geotechnical in situ exploration.

\begin{tabular}{|c|c|c|c|c|c|c|}
\hline Famille & Description & $\begin{array}{c}\text { Module } \\
\text { pressiométrique } \\
E_{\text {. }} \\
\text { (MPa) }\end{array}$ & $\begin{array}{l}\text { feart- } \\
\text { type } \\
\text { (MPa) }\end{array}$ & $\begin{array}{l}\text { Pression } \\
\text { limite } \\
\bar{P} \text { f } \\
\text { (MPa) }\end{array}$ & $\begin{array}{l}\text { Eicart- } \\
\text { type } \\
\text { (MPa) }\end{array}$ & $\begin{array}{l}\text { Nombre } \\
\text { d'essais }\end{array}$ \\
\hline F1 & Remblai de surface & 21,2 & 6,76 & 0,99 & 0,44 & 6 \\
\hline F3 & Limon sableux marron à vert de surface & 5,1 & 0,3 & 0,42 & 0,16 & 23 \\
\hline F4 & Sables et graviers & 37,8 & 26,7 & 3,6 & 1,63 & 60 \\
\hline F5 & Limon argileux gris & 2,46 & 0,65 & 0,31 & 0,05 & 5 \\
\hline F6 & Sable fins et limon gris noir & 27.7 & 16,3 & 1.76 & 0,64 & 118 \\
\hline F7 & Limon sableux gris noir profond & 14,7 & 10,1 & 0,85 & 0,31 & 49 \\
\hline F8 & Sable profond (sablon) compact & 40,2 & 18,2 & 2,8 & 0,51 & 14 \\
\hline
\end{tabular}


Le critère de Mohr-Coulomb caractérise la plasticité du sol avec un angle de frottement $\phi$ '. L'écoulement plastique non standard est déterminé par l'angle de dilatance $\Psi$. Le sol pulvérulent est considéré drainé.

\section{9 .9}

\section{Équilibre élastoplastique général}

Pour simplifier, nous ne détaillerons que le cas avec deux zones plastiques autour du pressiomètre. Alors, la relation générale d'équilibre entre contrainte et déformation au niveau du forage qui représente l'expression théorique pressiométrique est la suivante (Monnet et Khlif, 1994):

$$
\begin{aligned}
& \operatorname{Ln}\left[\frac{u_{a}}{a} \cdot(1+n)-C_{1}\right]=\delta \cdot \operatorname{Ln}(p)-\delta \cdot \operatorname{Ln}\left(\gamma_{1} z\right)+ \\
& \operatorname{Ln}\left[\left(1-K_{0}\right) \cdot \gamma \cdot z \frac{(1+n)}{2 \cdot G}-C_{1}\right] \\
& \text { avec: } \delta=\frac{1+n}{1-N} \text { et } C_{1}=\frac{n \cdot\left(\frac{u_{n}}{a}\right)(1+n)\left(\frac{\gamma \cdot z}{p}\right)^{s}+(1+n) \cdot\left(N-K_{0}\right) \cdot \frac{\gamma \cdot 2}{2 G}}{1+n\left(\frac{\gamma \cdot z}{p}\right)^{j}}(3)
\end{aligned}
$$

avec : $N=\left(1-\sin \phi^{\prime}\right) /\left(1+\sin \phi^{\prime}\right)$

et : $\mathrm{n}=(1-\sin \Psi) /(1+\sin \Psi)$

Ces formules sont obtenues en assurant, le respect de l'écoulement élastoplastique non standard dans les deux zones plastifiées, le respect de l'équilibre élastique dans la zone externe, la continuité de l'état de contrainte entre les différentes zones de comportement du sol autour de la sonde. La valeur de C. est très petite, et peut être négligée en première approximation. Dans ces conditions, la relation (2) montre qu'il existe une linéarité entre les logarithmes de la pression appliquée au forage et de la déformation radiale au forage. Cette linéarité avait déjà été trouvée précédemment (Hughes et al., 1977) mais sans définir la totalité de la courbe pressiométrique. La pente $\delta$ (3) de la relation linéaire est une fonction de l'angle de frottement interne $\phi^{\prime}$ et de l'angle de frottement intergranulaire $\phi_{\text {. }}$. La connaissance de $\phi_{\text {}}$ et de $\delta$ permet alors de déterminer directement l'angle de frottement interne.

\subsection{5}

\section{Pression limite conventionnelle}

Lorsque l'on atteint la pression limite, la cavité double de volume (après recharge jusqu'à une contrainte horizontale au repos), et la déformation radiale théorique devient alors égale à $\sqrt{2}-1$. Cette dernière valeur est introduite dans (2) ce qui permet de trouver alors la pression limite conventionnelle pour deux zones plastiques

$$
P_{\text {lcalcule é }}^{+}=\gamma \cdot z \hat{\frac{\left[(1+n) \cdot(\sqrt{2}-1)-C_{1}\right] 2 \cdot G}{\left[\left(1-K_{0}\right) \cdot(1+n) \cdot \gamma \cdot z-2 \cdot G \cdot C_{1}\right]}}
$$

Dans le cas de l'essai pressiométrique, le cisaillement principal se produit entre la direction de la contrainte radiale $\sigma_{\text {. }}^{\prime}$ et celle de la contrainte circonférentielle $\sigma_{\theta}{ }^{\prime}$ dans le plan horizontal. La contrainte verticale joue alors le rôle d'une contrainte de confinement du plan de cisaillement. Il est alors naturel que l'augmentation de la contrainte verticale se traduise, dans les sols frottants, par une augmentation proportionnelle de la résistance au cisaillement qui est mesurée ici par la pression limite (6). On trouve aussi que $\mathrm{p}$ est fonction du module de cisaillement $\mathrm{G}$, du rapport n qui est liée à la dilatance, du rapport $\mathrm{N}$ qui est lié à l'angle de frottement à travers le paramètre $\delta(2,4)$, mais aussi à l'angle de frottement intergranulaire $\phi_{\mu}(1,5)$.

\section{3}

\section{Corrélation entre la pression limite et l'angle de frottement}

Les nombreux essais réalisés au pressiomètre Ménard ont donné lieu à des études statistiques, ce qui a permis l'élaboration de nombreuses règles empiriques. Parmi celle-ci, nous avons retenu la corrélation reliant, pour les sols granulaires, la pression limite nette à l'angle de frottement interne (Amar, 1991). Cette relation s'écrit en unité de pression $\mathrm{kPa}$ :

$$
p_{i}^{*}=250.2^{\left[\left(\frac{0-24}{4}\right)\right]}
$$

Dans cette expression, le coefficient empirique 250 utilisable pour les sols de densité moyenne, peut être modulé entre 180 pour les sols lâches à 350 pour les sols structurés. Il est fonction du rapport $\mathrm{E}_{\mathrm{M}} / \mathrm{p}$. Nous utiliserons ici le coefficient moyen de la relation (7).

\section{5}

\section{Résultats des essais}

\section{1}

\section{Principe de la démarche expérimentale}

L'étude relative aux paramètres de cisaillement procède par étapes :

- mesure de l'angle de frottement intergranulaire $\phi_{u}$ à l'essai triaxial. On suppose que la nature géologique des grains du sol et leur état de surface sont indépendants de la granulométrie. Cette valeur est indépendante de l'état de densité du sol (Monnet et Gielly, 1978):

- mesure de la dilatance, puis de l'angle de frottement à l'essai pressiométrique. On suppose que le sol est non cohérent et parfaitement drainé. L'angle de frottement est alors considéré comme représentatif de l'état de densité local du sol in situ. L'essai pressiométrique est ici interprété comme un essai de cisaillement unique à un niveau de contrainte moyen imposé par le poids des terres au repos :

- lissage des valeurs de l'angle de frottement en fonction de la profondeur pour en déduire une valeur moyenne applicable à l'ensemble du projet. Les valeurs extrêmes des angles de frottement sont exclues car elles peuvent être dues à une cohésion du sol et à la granulométrie dont la résistance se reporte artificiellement sur la valeur du frottement. En effet, la présence éventuelle de gros cailloux à proximité de la sonde donnera une surestimation à la fois de la pression limite et de l'angle de frottement interne, la zone plastique au contact de la sonde n'existant plus. 


\section{Les essais triaxiaux consolidés et drainés}

Ces essais ont été réalisés au LIRIGM de l'université Joseph-Fourier à Grenoble sur des échantillons carottés non remaniés, mis à la pression d'essai, sous une contre pression de $100 \mathrm{kPa}$, le drainage étant ouvert pendant la période de consolidation. Les essais sont faits sur des échantillons de diamètre $7 \mathrm{~cm}$ et de hauteur $15 \mathrm{~cm}$ sans dispositif d'anti-frettage aux extrémités, selon la norme NF P 94-070 (1994). L'échantillon a été carotté à la dimension de l'éprouvette dans des prélèvements paraffinés venant du chantier. Il a été ensuite saturé et la pression latérale appliquée. Trois heures plus tard, la consolidation terminée, le cisaillement est réalisé à la vitesse de $0,06 \mathrm{~mm} / \mathrm{min}$ ce qui correspond à une durée d'essai de $10 \mathrm{~h}$ environ. Le drainage a été laissé libre pendant le cisaillement et la mesure de la variation du volume a été faite par l'intérieur de l'échantillon. Les résultats sont présentés sur les figures 6 et 7. Les caractéristiques physiques et mécaniques sont indiquées sur les tableaux II et III. Ces résultats ne sont exploités qu'en terme de frottement intergranulaire.

\section{Autres essais de cisaillement}

Des essais de cisaillement de type triaxiaux consolidés non drainés avec mesure de la pression interstitielle $(\mathrm{cu}+\mathrm{u})$ et de cisaillement direct drainé avec identification physique ont donné les résultats portés dans les tableaux 2 et 3.

\section{$5 \cdot 4$}

\section{Les essais triaxiaux cycliques}

Le tunnel est implanté en zone de sismicité IB. Cependant, une amplification locale de l'amplitude des ondes sismiques dans le bassin de Grenoble, pourrait amener un surclassement du site et de l'ouvrage. Pour étudier le risque de liquéfaction éventuelle des sols tels que les limons sableux, des essais cycliques ont été réalisés au CETE d'Aix. Ils montrent un fort coefficient de réduction du module élastique non drainé $E_{\mu}$ sous charge cyclique, en fonction du niveau de déformation. Une étude complémentaire par essais in situ au piézocône est envisagée.

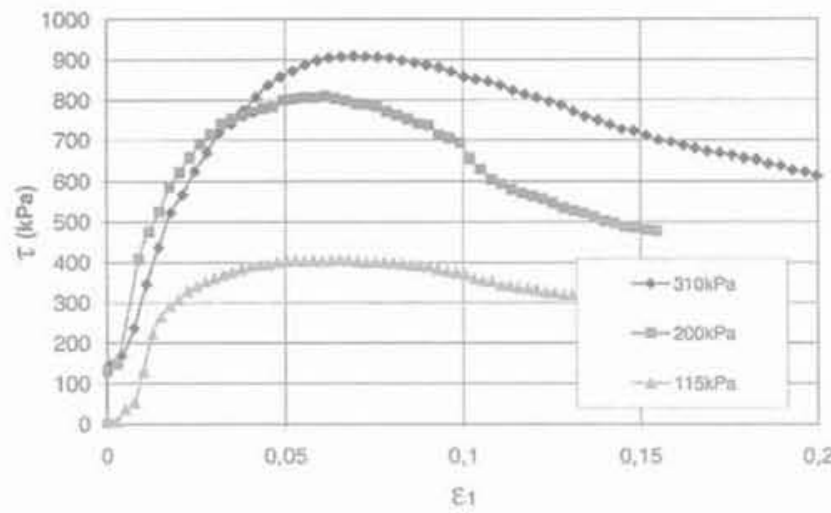

FG.6 Comportement au cisaillement du sol F7 pendant l'essai triaxial.

Shearing behaviour of the F7 soil on the triaxial test.

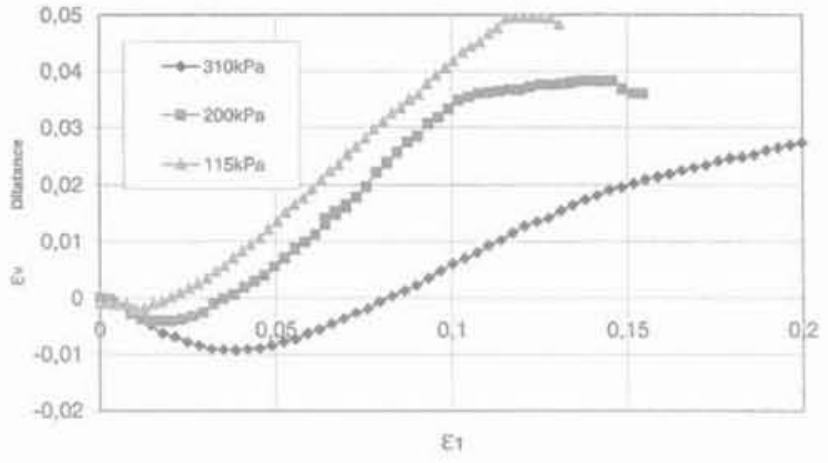

FF.7 Comportement en dilatance du sol F7 pendant l'essai triaxial.

Dilatancy behaviour of $\mathrm{F} 7$ soil on the triaxial test.

TABLEAUIII Les caractéristiques physiques mesurées au laboratoire.

The physical characteristics of the samples tested on laboratory.

\begin{tabular}{|c|c|c|c|c|c|c|c|}
\hline Famille & Description & $\begin{array}{l}\text { Nombre } \\
\text { échantillon }\end{array}$ & $\begin{array}{l}\text { Teneur } \\
\text { en eau } \%\end{array}$ & $\begin{array}{l}\text { Degré de } \\
\text { saturation }\end{array}$ & $\begin{array}{c}\text { Masse } \\
\text { volumique } \\
\text { sèche }(\mathrm{kg} / \mathrm{m})\end{array}$ & $\begin{array}{l}\% \text { de fines } \\
<80 \mu\end{array}$ & $\begin{array}{c}\text { Classification } \\
\text { GIR }\end{array}$ \\
\hline F6 & Sable fins et limon gris noir & 1 & 26 & 100 & 1640 & 50 & A1 \\
\hline F6 & Sable fins et limon gris noir & 12 & 23 & & 1650 & 18 & B2-B5 \\
\hline F7 & Limon sableux gris noir profond & 1 & 27 & 100 & 1703 & 4 & B1-B2 \\
\hline F7 & Limon sableux gris noir profond & 1 & 25 & 100 & 1656 & 16 & B5-B6 \\
\hline F7 & Limon sableux gris noir profond & 13 & 24 & & 1620 & 20 à 100 & $\mathrm{~A} 1-\mathrm{B} 5$ \\
\hline
\end{tabular}


TABLEAU III. Les caractéristiques mécaniques déduites des essais triaxiaux consolidés drainés. The mechanical results on consolidated drained triaxial test.

\begin{tabular}{|c|c|c|c|c|c|c|c|c|}
\hline Famille & Description & $\begin{array}{l}\text { Nombre } \\
\text { essais }\end{array}$ & $\begin{array}{l}\text { Mociule } \\
\text { d'Young } \\
\text { E (MPa) }\end{array}$ & $\begin{array}{c}\text { Coefficient } \\
\text { de Poisson } \\
v\end{array}$ & 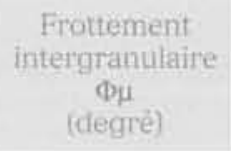 & $\begin{array}{l}\text { Cohésion } \\
\qquad \mathrm{C}^{\prime} \\
\text { (kPa) }\end{array}$ & $\begin{array}{l}\text { Frattement } \\
\text { interne } \\
\Phi \\
\text { (degré) }\end{array}$ & Rif \\
\hline F6 & Sable fins et limon gris noir & 1 & 36 & 0,265 & 29,8 & 0 & 36,4 & 0,847 \\
\hline F6 & Sable fins et limon gris noir & 7 & & & & 0 à 13,5 & 37,4 & \\
\hline$F 7$ & Limon sableux gris noir profond & 1 & 23,8 & 0,392 & 32,9 & 0 & 38,2 & 0,835 \\
\hline$F 7$ & Limon sableux gris noir profond & 1 & 17 & 0,388 & 30,2 & 0 & 36,4 & 0,696 \\
\hline FT & Limon sableux gris noir profond & 5 & & & & $\overline{0}$ & 35,2 & \\
\hline
\end{tabular}

\section{5}

\section{Essais pressiométriques}

\section{SI5.7 \\ Procédure expérimentale}

Les corrections classiques (NFP 94-110-1, 2000) sont appliquées aux pressions et volumes mesurés et les notations $\left(E_{\mathrm{M}}, \mathrm{E}, \mathrm{p}_{\mathrm{f}}\right)$ correspondent à des valeurs déterminées par la norme. Des corrections supplémentaires sont apportées aux pressions et volumes pour tenir compte, de la différence entre le rayon où la pression s'applique (à l'intérieur du tube fendu du pressiomètre lanterné battu) et celui où le sol réagit (à l'extérieur du tube fendu), de la déformée en poutre encastrée de la lanterne (Brevet Gaiatech, 1989) ou en parabole de la membrane (Fawaz et al. 2000), et de la distribution non uniforme de la pression le long de la sonde (Basudhar et Kumar, 1995). Les notations ( $\left.E_{M} ;, E^{*}, p_{-}^{-}\right)$avec l'exposant + correspondent à des valeurs calculées avec ces corrections supplémentaires.

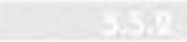

\section{Analyse pressiométrique}

Dans un premier temps, on mesure le module élastique G. La formule (2) montre que les courbes pressiométriques dépendent à la fois du module élastique et de T'angle de frottement interne $\phi^{\prime}$. Pour séparer l'influence de ces deux variables, il a été réalisé des essais à cycle qui permettent de déterminer indépendamment le module G (Fig. 9).

Dans un deuxième temps l'angle de frottement interne $\phi^{\prime}$ est mesuré par la pente moyenne $\delta$ de la relation linéaire (Fig. 8) entre les logarithmes des pressions et déformations radiales mesurées au forage, pour l'ensemble des points pour lesquels la pression dépasse la valeur du fluage. La valeur $r_{0}$ est le rayon initial du forage qui correspond au retour du terrain dans l'état initial avant forage. La valeur ainsi déterminée n'est qu'une approche sommaire du frottement et peut être remise en cause par la phase suivante de contróle des paramètres. Cette linéarité dépend du frottement intergranulaire $\phi_{u}$ mesuré sur les essais triaxiaux. L'effet lié au remaniement initial du sol par le forage préalable à l'essai est ici limité puisque l'interprétation bi-logarithmique utilise la partie de la courbe pressiométrique après le fluage.
Dans un troisième temps, le contrôle et l'ajustement définitif des caractéristiques mécaniques sont faits par la superposition des courbes expérimentales et thêoriques (Fig. 9), mais aussi par la comparaison (Tableau IV) des pressions limites expérimentales et théoriques (Monnet, 1990: Monnet et Khlif, 1994). Cette phase de l'interprétation est très importante, et on peut autoriser jusqu'à $17 \%$ d'écart en moyenne entre les valeurs des pressions limites théoriques et expérimentales. On procède d'abord par la vérification de la superposition des cycles charge-décharge pour valider le module de cisaillement, puis par la vérification de la superposition des parties terminales des courbes pressiométriques théoriques et expérimentales pour valider l'angle de frottement. Dans le cas où le frottement intergranulaire n'a pas pu être mesuré, on a pris forfaitairement la valeur moyenne des sols granulaires, soit $30^{\circ}$. Les résultats de l'analyse sont portés sur le tableau V. L'angle de frottement interne est ainsi déterminé. On remarque un écart-type de l'ordre de $1,5^{\circ}$ à $4^{\circ}$ pour toutes les familles de sol explorées (familles F3, F6, F7) ce qui traduit une bonne homogénéité. Par contre pour la famille F4 de sables et graves, l'écart-type sur les angles de frottement atteint $9^{\circ}$ qui provient de leur forte hétérogénéité naturelle pas du tout surprenante compte tenu de la variation de $p_{1}^{*}$,

Enfin, nous avons utilisé la formule (7) de corrélation donnée par Ménard. L'angle de frottement est calculé à partir de la valeur moyenne de la pression limite obtenue dans la couche. Les résultats sont portés dans le tableau $\mathrm{V}$. On remarque un accord à $1.5^{\circ}$ à $4^{\circ}$ près des déterminations du frottement par corrélation avec la détermination par la théorie présentée, pour les familles F4 (sables et graves) et F7 (limons sableux gris).

Par contre, il existe une différence de $5,2^{\circ}$ dans la couche F6 (sable et limon gris noir). On atteint dans cette couche les valeurs les plus basses possibles du frottement à $30^{\circ}$, proche de l'angle de frottement intergranulaire, par la théorie pressiométrique, alors que l'analyse corrélative donne une estimation de l'angle de frottement à $35,2^{\circ}$. La valeur trouvée à l'essai triaxial est de $37,3^{\circ}$ sur un échantillon reconsolidé. Il a été constaté cependant une perte de volume importante à l'intérieur de la carotte, lors de la phase de drainage et de consolidation à la mise en place de l'essai triaxial liée à un compactage important du sol. On peut donc penser que la valeur de frottement trouvée au laboratoire est surestimée et que l'on ne peut déduire de cet essai que $l^{\prime}$ angle de frottement intergranulaire, soit jci $30^{\circ}$ pour le sol F6. Néanmoins, c'est la valeur trouvée par l'approche corrélative qui a été retenue comme la valeur moyenne de référence pour cette famille. 


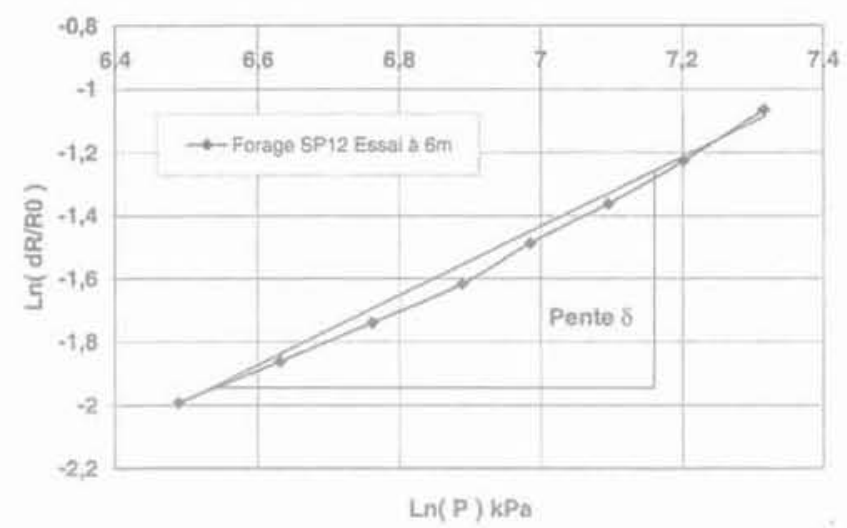

FIG. 8 Mesure de l'angle de frottement interne par la valeur de la pente de la relation linéaire entre les logarithmes des déformations et des pressions, Forage SP12 à $6 \mathrm{~m}$ de profondeur.

Measurement of the internal angle of friction by the slope of the linear relation between the logarithms of radial strain and stress, Borehole SP12 at 6 m depth.

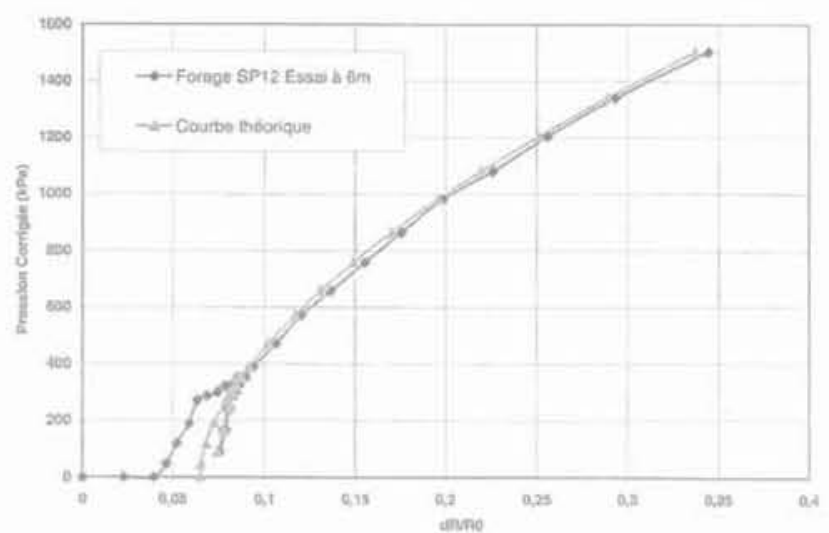

FIG.9 Contrôle des caractéristiques mécaniques, comparaison entre les courbes pressiométriques expérimentales et théoriques, Forage SP12 à $6 \mathrm{~m}$ de profondeur.

Control of the mechanical characteristics, comparison between theoretical and experimental pressuremeter curves, Borehole SP12 at $6 \mathrm{~m}$ depth.

TABtEAUIV Résultats de l'analyse pressiométrique.

Results of the pressuremeter analysis.

\begin{tabular}{|c|c|c|c|c|c|c|c|c|}
\hline Famille & Forage & $\begin{array}{c}\text { Profondeur } \\
\text { (m) }\end{array}$ & $\begin{array}{c}\mathrm{E}_{\mathrm{H}} \\
(\mathrm{MPa})\end{array}$ & (MPa) & $E_{\text {SM }} / E^{-}$ & $\mathrm{PP}^{+}$ & (kPa) & $\begin{array}{c}\phi \not \\
\text { (Alegré) }\end{array}$ \\
\hline $\begin{array}{l}\mathrm{F} 3 \\
\mathrm{~F} 4 \\
\mathrm{~F} 4 \\
\mathrm{~F} 6 \\
\mathrm{~F} 6 \\
\mathrm{~F} 6\end{array}$ & SP 5 & $\begin{array}{c}4 \\
6 \\
8 \\
13 \\
16 \\
18 \\
\end{array}$ & $\begin{array}{r}7,9 \\
7,7 \\
15,9 \\
15,2 \\
16,7 \\
27,5\end{array}$ & $\begin{array}{l}22,7 \\
18,3 \\
50 \\
31,3 \\
30,2 \\
42,7\end{array}$ & $\begin{array}{l}0,35 \\
0,42 \\
0,32 \\
0,48 \\
0,55 \\
0,64\end{array}$ & $\begin{array}{r}590 \\
1640 \\
3780 \\
810 \\
685 \\
760\end{array}$ & $\begin{array}{r}700 \\
1550 \\
3240 \\
975 \\
1010 \\
1130\end{array}$ & $\begin{array}{l}36 \\
52 \\
53 \\
31 \\
30 \\
28\end{array}$ \\
\hline $\begin{array}{l}\text { F3 } \\
\text { F4 } \\
\text { F4 } \\
\text { F6 } \\
\text { F6 } \\
\text { F7 } \\
\text { F7 } \\
\text { F7 } \\
\text { F8 }\end{array}$ & SP 6 & $\begin{array}{c}3 \\
6 \\
9 \\
12 \\
15 \\
18 \\
21 \\
24 \\
28\end{array}$ & $\begin{array}{c}2,2 \\
4,5 \\
7,3 \\
13,4 \\
11,2 \\
6,2 \\
31 \\
18,6 \\
3,3\end{array}$ & $\begin{array}{c}4,6 \\
17,5 \\
7,8 \\
28,2 \\
31,3 \\
18 \\
54,3 \\
40,7 \\
7\end{array}$ & $\begin{array}{l}0,48 \\
0,26 \\
0,93 \\
0,47 \\
0,36 \\
0,34 \\
0,57 \\
0,46 \\
0,47\end{array}$ & $\begin{array}{r}270 \\
440 \\
735 \\
795 \\
860 \\
760 \\
920 \\
1335 \\
540\end{array}$ & $\begin{array}{r}290 \\
590 \\
735 \\
905 \\
1055 \\
1330 \\
1325 \\
1515 \\
870\end{array}$ & $\begin{array}{l}33 \\
32 \\
32 \\
31 \\
31 \\
39 \\
28 \\
31 \\
30\end{array}$ \\
\hline $\begin{array}{l}\text { F4 } \\
\text { F4 } \\
\text { F4 } \\
\text { F6 } \\
\text { F6 }\end{array}$ & SP 8 & $\begin{array}{l}9 \\
13 \\
17 \\
24 \\
29\end{array}$ & $\begin{array}{l}13,1 \\
11,6 \\
17,7 \\
90,1 \\
29,3\end{array}$ & $\begin{array}{c}42,3 \\
30 \\
41 \\
118,8 \\
98\end{array}$ & $\begin{array}{l}0,31 \\
0,39 \\
0,43 \\
0,76 \\
0,30\end{array}$ & $\begin{array}{r}1030 \\
985 \\
1055 \\
1300 \\
1500\end{array}$ & $\begin{array}{l}1190 \\
1105 \\
1150 \\
1620 \\
1790\end{array}$ & $\begin{array}{l}36 \\
34 \\
30 \\
26 \\
26\end{array}$ \\
\hline $\begin{array}{l}\mathrm{F} 4 \\
F 6 \\
\mathrm{~F} 6 \\
\mathrm{~F} 6\end{array}$ & SP 9 & $\begin{array}{l}13 \\
19 \\
24 \\
28 \\
\end{array}$ & $\begin{array}{r}9,3 \\
53,2 \\
39,4 \\
9 \\
\end{array}$ & $\begin{array}{r}100 \\
40 \\
50 \\
1000 \\
\end{array}$ & $\begin{array}{l}0,09 \\
1,00 \\
0,79 \\
0,09 \\
\end{array}$ & $\begin{array}{r}2985 \\
835 \\
1135 \\
1655 \\
\end{array}$ & $\begin{array}{l}3350 \\
1095 \\
1500 \\
1525 \\
\end{array}$ & $\begin{array}{l}44 \\
30 \\
30 \\
30 \\
\end{array}$ \\
\hline $\begin{array}{l}F 6 \\
F 6 \\
F 6 \\
F 6 \\
F 6 \\
\end{array}$ & SP 10 & $\begin{array}{l}15 \\
19 \\
23 \\
27 \\
32 \\
\end{array}$ & $\begin{array}{l}13 \\
12,9 \\
21 \\
16,7 \\
32,2\end{array}$ & $\begin{array}{c}35 \\
48,2 \\
62,1 \\
58,2 \\
126,2\end{array}$ & $\begin{array}{l}0,37 \\
0,27 \\
0,34 \\
0,12\end{array}$ & $\begin{array}{r}945 \\
980 \\
1420 \\
1320 \\
1900 \\
\end{array}$ & $\begin{array}{l}1105 \\
1240 \\
1700 \\
1700 \\
2425\end{array}$ & $\begin{array}{l}31 \\
30 \\
31 \\
30 \\
30\end{array}$ \\
\hline $\begin{array}{l}\text { F4 } \\
\text { F4 } \\
\text { F6 } \\
\text { F6 } \\
\text { F6 } \\
\text { F6 }\end{array}$ & SP11 & $\begin{array}{l}15 \\
19 \\
22 \\
25 \\
28 \\
31 \\
\end{array}$ & $\begin{array}{c}12 \\
13 \\
8,1 \\
8,3 \\
6,9 \\
21.4 \\
\end{array}$ & $\begin{array}{l}37,2 \\
43,2 \\
20 \\
33,9 \\
26 \\
83 \\
\end{array}$ & $\begin{array}{l}0,32 \\
0,30 \\
0,40 \\
0,24 \\
0,03 \\
0,26\end{array}$ & $\begin{array}{r}2565 \\
980 \\
1185 \\
1230 \\
1310 \\
1705 \\
\end{array}$ & $\begin{array}{l}2560 \\
1430 \\
1285 \\
1480 \\
1375 \\
1965 \\
\end{array}$ & $\begin{array}{l}48 \\
32 \\
35 \\
32 \\
31 \\
30 \\
\end{array}$ \\
\hline $\begin{array}{l}\text { F4 } \\
\text { F4 } \\
\text { F4 } \\
\text { F4 } \\
\text { F6 } \\
\text { F6 } \\
\text { F6 } \\
\text { F6 } \\
\text { F6 }\end{array}$ & SP 12 & $\begin{array}{l}3 \\
6 \\
8 \\
12 \\
16 \\
20 \\
22 \\
25 \\
28 \\
\end{array}$ & $\begin{array}{r}15,1 \\
8,8 \\
23,4 \\
10,3 \\
29,4 \\
4,9 \\
7,8 \\
23 \\
24,6\end{array}$ & $\begin{array}{l}44,8 \\
31,5 \\
64,2 \\
38,7 \\
67,5 \\
37,9 \\
40,6 \\
90,9 \\
93,6\end{array}$ & $\begin{array}{l}0,34 \\
0,28 \\
0,36 \\
0,27 \\
0,44 \\
0,13 \\
0,19 \\
0,25 \\
0,26\end{array}$ & $\begin{array}{r}1790 \\
1725 \\
3010 \\
1125 \\
830 \\
935 \\
1135 \\
1240 \\
1825\end{array}$ & $\begin{array}{r}2220 \\
1920 \\
3530 \\
1140 \\
980 \\
1220 \\
1285 \\
1525 \\
1875\end{array}$ & $\begin{array}{l}52 \\
50 \\
52 \\
34 \\
24 \\
30 \\
30 \\
26 \\
30\end{array}$ \\
\hline
\end{tabular}




\section{Conception du tunnel sous l'lle verte}

6.1

\section{Synthèse du contexte géotechnique et hydrogéologique, et choix des hypothèses géotechniques}

Le contexte géotechnique du sous-sol du quartier de l'Tle verte et des Sablons peut ètre schématisé en suivant le projet de l'est (secteur Sablons) vers l'ouest (secteur Bastille) :

- à l'est, côté Sablons, un alluvionnement de sables (sablon) et sables et graviers compacts avec quelques lentilles limoneuses plus làches, pour le tube Sud sur $66 \%$ du tracé, pour le tube nord sur $80 \%$ du tracé : - à l'ouest, còté Bastille, la transition à des sables limoneux, puis des limons sableux à passages infradécimétriques argileux se fait progressivement sur le reste du tracé.

Les sables et limons sont homométriques, potentiellement liquéfiables sous faibles contraintes verticales. Les limons ont une structure variée, avec des niveaux sableux intercalés et des passages peu consolidés, notamment au contact du rocher constituant la base orientale du Rabot quand le projet passe l'Isère en profondeur. Ce sous-sol est recouvert d'une couche superficielle de remblai et limons peu compacts d'épaisseur 3 à $5 \mathrm{~m}$.

Tous ces sols sont totalement baignés par une nappe en relation avec la boucle de l'Isère s'écoulant dans la direction est-ouest. La perméabilité est décroissante d'est en ouest. La synthèse géotechnique par familles de sols est indiquée dans le tableau V.

La synthèse par famille, réalisée à partir des essais pressiométriques a fait l'objet d'une étude statistique définissant, la valeur moyenne $\bar{X}$, l'écart-type estimé $\sigma_{n}$, la valeur caractéristique X en prenant en compte l'incertitude fonction du nombre d'essais $\mathrm{N}$ et la probabilité $\beta$ que la valeur d'une mesure X soit inférieure à la valeur caractéristique $X$. Dans cette étude statistique, on retient $\beta=5 \%$. La valeur caractéristique est alors :

$$
\mathrm{X}_{\mathrm{k}}=\mathrm{k}_{\mathrm{D}}\left(\overline{\mathrm{X}}-\mathrm{k}_{\mathrm{N}} / \mathrm{k}_{\mathrm{y}}, \sigma_{\mathrm{n}}\right)
$$

avec : $k_{0}=1$ qui prend en compte la représentativité des données; $\mathrm{k}_{\mathrm{v}}=\sqrt{5}$ pour le tunnel de grand diamètre, qui prend en compte la réduction de la variance en fonction du volume de sol intéressé par le phénomène de tassement ou de rupture;

$\mathrm{k}_{\mathrm{N}}$ dont la valeur est lue dans les tables de probabilité, qui prend en compte le niveau de risque retenu et l'incertitude de statistique liée au nombre de mesures $\mathrm{N}$.

Cependant, des incertitudes sur les sols subsistent et notamment :

- la rencontre des lentilles décimétriques de sols compressibles (tourbes, vases ou argiles) dans le dépôt de sable, trouvées dans deux sondages carottés profonds réalisés pour l'étude des fondations des tours de l'Isère

- la présence d'obstacles naturels tels que blocs ou troncs d'arbre.

Les valeurs $\mathrm{X}_{\mathrm{k}}$ caractéristiques pour chaque famille de sol et par type de mesures sont portées dans le tableau VI.

\section{Conséquences du contexte du site sur la conception du tunnel, au stade de l'avant-projet}

Le projet de tunnel doit prendre en compte non seulement les données du contexte géotechnique, mais aussi celles du site urbain avec ses propres contraintes de sensibilité au tassement et de risque vis-à-vis du bâti. Cet examen d'ensemble des données d'entrée du projet permet de préciser son calage en tracé en plan et en profil en long, en estimant au mieux dès ce stade de l'étude les conséquences de la construction de l'ouvrage sur le site de surface en relation avec les méthodes envisagées suivant une démarche établie par Chapeau et Schwenzfeier (1987).

\section{QSin}

\section{Contexte géotechnique et choix des méthodes}

Le tunnel sera totalement réalisè en terrain meuble et aquifère nécessitant l'utilisation d'un bouclier avec pressurisation du front de taille. La pressurisation à la boue, bien adaptée au cas des sables et graviers et des

TABLEAUV Synthèse par famille de sol des résultats de la reconnaissance géotechnique. Synthesis by soil Family of the geotechnical campaign.

\begin{tabular}{|c|c|c|c|c|c|c|c|c|}
\hline Famille & ${ }_{(\mathrm{MPa})}^{\mathrm{P}}$ & $\frac{\mathrm{E}_{\mathrm{M}}}{(\mathrm{MPa})}$ & $\frac{E}{(M P a)}$ & $\alpha$ & $\begin{array}{c}\Phi^{\prime} \\
\text { Corrélation } \\
\text { (degré) }\end{array}$ & $\begin{array}{l}\text { Ecant-type } \\
\text { (degré) }\end{array}$ & $\begin{array}{l}\Phi^{\prime} \\
\text { théorique } \\
\text { (degre) }\end{array}$ & $\begin{array}{l}\text { Ecant-type } \\
\text { (degrê) }\end{array}$ \\
\hline F1 & 0,99 & 21,2 & 31,6 & 0,67 & 31.9 & 2.1 & & \\
\hline F3 & 0,42 & 5,1 & 7,6 & 0,67 & 27 & 1.9 & 34,5 & 1.5 \\
\hline $\mathrm{F} 4$ & 3.6 & 37.8 & 114,5 & 0,33 & 39.4 & 2,2 & 41,5 & 9 \\
\hline F5 & 0,31 & 2,46 & 12,1 & 0,5 & 25,2 & 0,7 & & \\
\hline F6 & 1,76 & 27,7 & 55,4 & 0,50 & 35,3 & 1,8 & 30 & 2 \\
\hline$F 7$ & 0,85 & 14.7 & 21,9 & 0.67 & 31.1 & 1,8 & 32,6 & 4 \\
\hline F8 & 2,8 & 40,2 & 121,8 & 0,33 & 37.9 & 1 & & \\
\hline
\end{tabular}


TABLEAU V Valeurs caractéristiques des paramètres géotechniques.

Characteristic values for geotechnical parameters.

\begin{tabular}{|c|c|c|c|c|c|c|c|}
\hline Famille & Description & $\frac{\mathrm{p}^{*}}{(\mathrm{MPa})}$ & $\underset{\text { (MPa) }}{\mathrm{E}_{\mathrm{M}}}$ & $\mathrm{E}_{M} / \mathrm{p}^{*}$ & $\alpha$ & $\frac{E}{(M P a)}$ & $\begin{array}{c}\Phi \\
\text { (degré) }\end{array}$ \\
\hline $\mathrm{F} 1$ & Remblai de surface & 0,65 & 16 & 24,6 & 0.67 & 24 & 29 \\
\hline F3 & Limon sableux marron à vert de surface & 0,3 & 3 & 10 & 0,67 & 4.5 & 25 \\
\hline F4 & Sables et graviers & 2,4 & 18 & 7,5 & 0,33 & 55 & 37 \\
\hline F5 & Limon argileux gris & 0,3 & 1,9 & 6,3 & 0,5 & 3,8 & 25 \\
\hline F6 & Sable fins et limon gris noir & 1,3 & 16 & 12,3 & 0,5 & 30 & 33 \\
\hline$F 7$ & Limon sableux gris noir profond & 0,6 & 7,2 & 12 & 0,67 & 11 & 29 \\
\hline F8 & Sable profond (sablon) compact & 2.4 & 26,4 & 11 & 0,33 & 80 & 37 \\
\hline
\end{tabular}

sables légèrement limoneux est à étudier spécifiquement pour les limons avec une adaptation de la nature de la boue par ajouts de polymère. II n'y a pas de risque, a priori, de pertes brutales de boue et donc de dépressurisation du front de taille, du fait des perméabilités mesurées $\left(\mathrm{k}<10^{-3} \mathrm{~m} / \mathrm{s}\right)$, du pourcentage élevé de sable fin et de l'absence de vides dans ce dépôt continu. Ces terrains à forte proportion de sables fins et limoneux sont aussi bien adaptés à l'utilisation d'un bouclier à pression de terre mais d'autres phénomènes comme la portance dans les limons mous restreignent cette possibilité.

En regard du marinage des déblais, la granulométrie de tous les sols permet le transport hydraulique. La présence d'éléments très fins voire d'argile conduira localement à un traitement approprié, avec réduction des cadences d'avancement.

En ce qui concerne la propulsion du bouclier, elle peut être rendue problématique par la présence, à l'ouest :

- des limons en radier rendant délicate la conduite du tunnelier. Celui-ci aura d'autant plus tendance à piquer du nez que son centre de gravité sera déplacé vers Y'avant (cas pour les boucliers à pression de terre). Ce guidage plus difficile avec enfoncement du tunnelier puis rattrapage est source de "perte de terrains $)$ et donc de tassement en surface:

- du rocher sous I'Tsère qui pourrait bloquer sa progression, à moins, soit d'équiper et de concevoir le bouclier pour travailler au rocher (peu compatible avec un bouclier en terrain meuble maitrisant les tassements), soit de transformer par minage préalable le rocher en terrain « meuble $n$ (solution a priori possible).

La présence d'obstacles naturels reste un risque à prendre en compte dans le choix des techniques de pressurisation (accès au front) ou de tracé (accès depuis la surface).

Le revêtement du tunnel sera constitué d'anneaux de voussoirs préfabriqués en béton mis en place à l'abri de la jupe du bouclier. La nature pulvérulente des terrains immergés rend obligatoire le remplissage continu du vide annulaire si l'on veut limiter les tassements en site urbain. La présence de la nappe impose des voussoirs assemblés par boulons et équipés de joints d'étanchéité. La conception et le dimensionnement du revêtement ne posent pas de problème particulier.

Vis-à-vis du séisme, les sols sont potentiellement liquéfiables (sable homométrique, limon sableux), mais ce risque est limité si localement la pression intersti- tielle générée par l'action sismique ne peut pas se dissiper (cas des radiers plats) et si la contrainte verticale est faible (cas des tunnels à faible profondeur soumis à la poussée d'Archimède). Ce risque est par contre accentué avec un grand diarnètre du tunnel. Le potentiel de liquéfaction est aussi préjudiciable à la progression du tunnelier. En effet, celui-ci génère des vibrations notamment en terrains hétérogènes, de granulométrie grossière, pouvant produire des surpressions interstitielles autour ou devant le tunnelier si le déconfinement est important. Ce phénomène ne sera pas prépondẻrant si la maîtrise de la pressurisation est bonne et celle du guidage est effective.

Tous ces éléments conduisent à retenir, à ce stade de l'étude, une pressurisation par boue liquide, régulée par une bulle d'air comprimé, de manière à maintenir parfaitement et à tout moment la stabilité du front de taille.

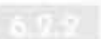

\section{Prise en compte du site sur le creusement au bouclier}

La rocade Nord se situe dans un contexte urbain dense et pose plusieurs difficultés techniques dont cinq particulièrement importantes, les franchissements sous-fluviaux de l'Tsère autour de l'Tle verte, le passage de l'tle verte, l'alternance des formations géologiques, le franchissement sous-fluvial de l'Isère au niveau de la Porte de France, l'écoulement de la nappe alluviale dans la presqu'île.

Franchissement de l'Isère autour de l'lle verte

Le site des Sablons est très favorable à l'installation du chantier et à l'exécution d'un puits de démarrage du tunnelier car c'est une zone non urbanisée, d'accès facile, en terrain sablo-graveleux compacts en profondeur et présentant des limons de surface peu perméables. On attaquera ensuite la traversée sous-fluviale de l'Isère qui pourrait être réalisée, soit en immergeant des caissons préfabriqués au fond d'une souille, soit en creusant suffisamment profondément sous le cours d'eau au moyen d'un tunnelier assurant un soutènement latéral et frontal des terres. La technique du tunnelier à pression de boue a donc été choisie dans cette traversée. Elle permet une continuité du chantier avec la section de traversée souterraine de l'tle verte qui doit se faire obligatoirement par cette technique. Comme les terrains sous-jacents sont constitués de sables fins et sables et graviers audessus, un approfondissement du profil en long et avec une bonne maitrise du tunnelier pourrait permettre de réduire les tassements et les risques de fontis. 


\section{Le passage de l'Ile verte}

Le passage de l'lle verte se fait d'est en ouest sous un bâti léger sans contraintes particulières et avec des terrains sablo-graveleux propices, puis le site urbain devient très sensible avec des immeubles de grande hauteur et la présence d'une structure sur poteaux élancés ( église ») avec fondations sur pieux. Dans cette zone on a pu observer des tassements de surface de 2 à $8 \mathrm{~cm}$ par le décalage des trottoirs par rapport au bâti qui est fondé, a priori, sur les graviers sous-jacents. Le tassement est celui des limons de surface, généré par variation du niveau de la nappe. On note également un bâti constitué d'immeubles de hauteur moyenne à l'approche des limons en sous-sol. Il convient d'approfondir le profil en long pour élargir la cuvette de tassement et diminuer le tassement différentiel.

\section{Alternance de formations géologiques}

Le projet traverse successivement trois terrains distincts, les alluvions mêlées du Drac et de l'Isère à l'ouest, les marnes et calcaires du rocher de la Bastille au centre, les alluvions de l'Isère à l'est. En raison des traversées sous-fluviales de l'Isère et de l'écoulement de la nappe dans la presqu'ille, la technique du creusement au tunnelier à pression de boue est plus adaptée pour les extrémités du projet dans les alluvions. Pour traverser les marnes et calcaires du rocher de la Bastille, les techniques traditionnelles et le tunnelier sont adaptés. Cependant, le tunnelier à pression de boue doit faire l'objet de modifications substantielles du bouclier, du confinement, du système de marinage, pour pouvoir attaquer la roche après avoir creusé les alluvions. Il faut donc prévoir des chambres aux interfaces entre le rocher et les alluvions pour intervenir sur la machine ou réaliser une attaque traditionnelle. Ces chambres sont réalisées à l'aide de puits temporaires.

Le tracé reste à caler après une reconnaissance plus fine du toit du rocher et le choix définitif du profil en long, en intégrant les rayons minimaux à respecter tant du point de vue technique (bouclier) que fonctionnel (visibilité).

Le passage de l'Isère demande une étude spécifique, car il faut une bonne connaissance de la côte du fond de son lit, la possibilité de traiter par injection les terrains de couverture au-dessus du rocher et la possibilité de minage du rocher sans risque pour l'environnement (dégradation sur le bàti existant).

A l'interface ouest, pour la variante « Vercors $»$, le puits sera situé au niveau de la rue Durand-Savoyat.

L'abaissement du profil en long et son calage restent l'enjeu du projet d'un point de vue économique, technique et environnemental.

\section{La traversée de l'Isère au niveau de la Porte de France}

Dans le cas de la variante "Vercors »), la réalisation d'une traversée sous-fluviale à l'aide de caissons préfabriqués et immergés pose les mêmes difficultés techniques de gestion du risque de crue de la rivière qu'au droit de l'tle verte. De plus, elle implique la réalisation dans la presqu'île de parois moulées dont l'effet de barrage est décrit ci-après. Cette solution n'a donc pas été retenue.

La traversée de l'Isère à cet endroit peut être réalisée à l'aide d'un tunnelier ou en technique traditionnelle. En raison d'une remontée des marnes du rocher de la Bastille à la limite de l'Isère, un projet de franchis- sement sous-fluvial à cet endroit doit plonger profondément pour éviter de multiplier les interfaces entre le rocher et les alluvions.

Dans la variante $\propto$ Esplanade $»$ le franchissement de l'Isère se fait par un viaduc et ne présente que des difficultés liées à la fondation de l'ouvrage sur un socle rocheux dont le toit reste encore à préciser.

\section{Écoulement de la nappe alluviale dans la presqu'île}

L'Isère et le Drac encadrent la presqu'île, et la nappe associée s'écoule parallèlement à ces deux cours d'eau. Une couche de terrain imperméable se situe à une vingtaine de mètres sous le terrain naturel. Dans Thypothèse où la rocade nord passerait dans la presqu'île entre des parois moulées, leur profondeur créerait un barrage à l'écoulement de la nappe susceptible d'entraîner des tassements à l'aval et des désordres dans les fondations et les caves des bảtiments à l'amont. Pour prévenir de tels désordres, il faudrait rétablir l'écoulement en forant de nombreuses traversées dans les parois. Le coût de l'étude et de la réalisation de cette continuité hydraulique est très élevé. La réalisation de parois moulées dans la presqu'île a donc été rejetée.

La construction de tranchées couvertes dont le radier est implanté au niveau du toit de la nappe permet d'éviter l'effet de barrage associé. Elle présente également l'avantage de limiter à proximité du Laboratoire d'électronique et d'instrumentation (LETI) la formation et la propagation de vibrations dans le sol, tant en phase de travaux qu'en phase d'exploitation. Cette technique a été retenue pour la traversée du CENG.

Pour la variante «Vercors », la solution du tunnel creusé permet de plonger suffisamment vite, de limiter ainsi la section comprise dans la nappe et de s'affranchir de l'effet de barrage.

\section{ans 3}

\section{Synthèse et conclusions sur le choix des techniques de creusement et sur le projet}

Les contraintes fortes liées à l'environnement géotechnique de ce projet sont :

- la présence de la nappe qui nécessite un bouclier à front pressurisé ;

- le passage sous un bâti qui est sensible aux tassements, et qui nécessite une maîtrise parfaite de la pression de confinement, du guidage du tunnelier et du comblement en continu du vide annulaire ;

- la zone de graviers pratiquement rencontrée sur tout le tracé qui peut engendrer des vibrations pendant l'abattage et l'avancement du tunnelier et induire des phénomènes de liquéfaction des limons peu compacts et sables fins, avec perte de portance de la tête du tunnelier. Pour réduire, voire annihiler ces phénomènes liés, l'avancement du bouclier doit être accompagné d'un ajout de lubrifiant ;

- la présence de limons plus lâches en radier est un inconvénient pour le tunnelier à pression de terres, plus lourd et plus difficile à guider dans ce contexte à cause de sa tendance à plonger et qui induit par son principe des actions vibratoires plus importantes ; - la présence possible d'obstacles naturels (tels blocs erratiques et surtout troncs d'arbre fossilisés) nécessite 
l'intervention sous hyperbare dans la chambre d'abattage (comme pour le changement des outils) avec confinement du front de taille en toute sécurité pour les intervenants au front et vis-à-vis des incidences en surface.

En conclusion, vis-à-vis des conditions de terrains, les boucliers à pression de boue sont recommandés. Vis-à-vis des conditions et des contraintes de site, actuellement le bouclier à pression de boue avec régulation de la pression au front par bulle d'air répond le mieux techniquement au problème posé. L'utilisation d'un bouclier de grand diamètre représente cependant d'autres contraintes liées à son encombrement et son poids, montage, transport en site urbain, transfert qu'il s'agit d'examiner dès l'APS pour garantir la faisabilité du projet:

L'implantation du puits d'entrée côté Sablons et des installations de chantier est recommandée. Par contre, le puits de sortie, a priori à concevoir en rive droite de I'Isère, nécessite une étude spécifique pour rechercher son implantation optimale conditionnée par le contact sol/rocher, l'encombrement de surface, la réduction de largeur de l'Isère à compenser. C'est un point critique du projet qui engage la qualité du tracé voire sa faisabilité.

Le risque de tassement existe tout au long du tracé avec cependant des conditions géotechniques favorables là où les conditions du site sensible sont les plus fortes. La recherche de tracé au niveau des rues sans encombrement de la surface limite les risques vis-à-vis du bâti et rend accessible le front en cas d'incident grave nécessitant une intervention éventuelle depuis la surface, pour traitement spécifique.

\section{Conclusion}

Les reconnaissances pour le tunnel de la rocade nord de Grenoble ont privilégié les essais in situ, et en particulier l'essai pressiométrique qui a été utilisé avec un cycle de déchargement-rechargement, En effet, les essais de laboratoire sont considérés ici comme peu représentatifs des conditions de densité, de structuration et de consolidation du sol en place, car il a été constaté un remaniement important des échantillons, même avec les meilleures techniques de prélèvement. Les essais au laboratoire ont donc été considérés comme des essais d'identification physiques et mécaniques permettant d'obtenir les caractéristiques minimums sur lesquelles on peut compter, mais pas les conditions exactes du site.

L'essai pressiométrique a permis d'atteindre les caractéristiques de cisaillement et de frottement du sol en place avec une procédure spécifique.

L'analyse géotechnique du site du tunnel, montre une variabilité des caractéristiques mécaniques, prise en compte par le choix de valeurs minimales caractéristiques, $95 \%$ des valeurs mesurées étant supérieures à celles-ci. Il reste cependant des incertitudes liées notamment au risque de liquéfaction, la présence de limons lâches pouvant déstabiliser la tête du tunnelier, la présence d'obstacles éventuels de gros diamètre dans les paléochenaux et l'implantation du puits de sortie à l'ouest du projet avec un contact sol-rocher à préciser.

Ces incertitudes seront levées par des campagnes complémentaires de forages, sondages et de mesures géophysiques.

\section{Bibliographie}

Amar S., Clarke B.G.. Gambin M., Orr I.L.L. - i The application of pressuremeter test results to foundation design in Europe 1. European Regional Technical Committee $n^{\circ} 4$, Pressuremeter. A.A. Balkema, 1991, p. 1-23.

Baquelin F, Jezequel J.-F, Shields D.H. * The pressuremeter and foundation engineering y. Trans Tech Publications. 1978.

Basudhat P., Kumar D. - w Performance studies of cavity expansometer. A monocell pressuremeter. "The pressuremeter and its new avenues" 1 . Proc . 4th Int. Symp. Sherbrooke, 1995, p. 73-80.

Chapeau C., Schwenzfejer A. - $\approx$ Reconnaissance du sous-sol et creusement au bouclier: spécificitè des terrains meubles en site urbain в. AFTES C.R. des journées d'études internationales de Bordeaux, 1987.

Clarke B.G., Gambin M. - « Pressuremeter testing in onshore ground investigations i. ISSMGE Committee TC16, 1st Int. Cong. on site Characterisation.
Atlanta, Ed. Balkerna, 1998.

Fascicule 62, titre V-Règles technigues de conception et de calcul des fondations des ouvrages de génje civil, Cahier des clauses techniques générales appllcables aux marchés publics de travaux Circulaire $n^{\circ} 93-66$ du ministère de 1'Equipement, des Transports et du Logement, 1993.

Fawaz A., Biguenet G., Boulon M. - - Défor mations d'un sol pulvérulent lors de l'essai pressiométrique x. Revue française de géotechnique, $n^{\circ} 90,2000$, p. 3-13.

Gaiatech - Procédé d'essai de forage. Brevet français $n^{\circ} 8909674$, Lyon, 1989.

Gambin M. - « Vingt ans d'usage du pressiomètre en Europe 1. Congrès européen de Mécanique des sols et des travaux de fondation. Brighton, 1979.

Hughes J.M.O., Wroth C.P., Windle D. a Pressureter tests in sand 0. Geotechnique, vol. $27, \mathrm{n}^{\circ} 4,1977$, p. $455-477$.

Ménard L. - Pressiomètre Brevet français $n^{\circ}$ 1.117.983, Paris, 1955
Ménard L. - Mesures des propriétés physiques des sols 3 . Annales des Ponts et Chaussées, Paris, $n^{\circ} 14,1957$, p. $357-377$.

Monnet I Gielly \& - « Détermination d'une loi de comportement pour le cisaillement des sols pulvérulents $\%$. Revue française de géotechnique, $n^{\circ} 7,1978$, p. 45-66.

Monnet J. - " Theoretical study of elastoplastic equilibrium around pressuremeter in sands i. 3 th International Symposium on Pressuremeters, Oxford 1990 p. 137-148.

Monnet J. Khlif J. - $\pi$ Etude théorique et expérimentale de l'équilibre élastoplastique d'un sol pulvérulent autour du pressiomètre m. Revue française de géotechnique, $n^{\circ} 67,1994, \mathrm{p}, 3-12$

Norme française NF P 94-070 - Essai à l'appareil triaxial de révolution. AFNOR, 1994.

Norme francaise NF P 94-110-1 - Essai pressiométrique Ménard, Partie 1 : Essai sans cycle. AFNOR, 1999.

Norme française NF P 94-110-2 - Essai pressiométrique Ménard, Partie 2 : Essai avec cycle. AFNOR, 2000. 